\title{
Effect of surface tension on the adhesion between a rigid flat punch and a semi-infinite neo-Hookean half-
}

space

Tianshu Liu ${ }^{1}$, Anand Jagota ${ }^{2}$, Chung-Yuen Hui ${ }^{1 *}$

${ }^{1}$ Field of Theoretical \& Applied Mechanics, Dept. of Mechanical and Aerospace Engineering, Cornell University,

${ }^{2}$ Department of Chemical \& Biomolecular Engineering and Bioengineering Program, Lehigh University (USA)

*Corresponding Author, ch45@cornell.edu

Abstract

We study the effect of surface stress on theretraction of a rigid flat circular punch adhering to an incompressible neo-Hookean half spaceusing a large deformation finite element model (FEM). The effect of surface stress is determined by the dimensionless elasto-capillary number, $\omega=\sigma /(E a)$, where $\sigma$ is the isotropic solid-air interface surface stress which we shall called surface tension, $E$ is the small strain Young's modulus and $a$ is the radius of punch. Adhesion of the punch to the half space is characterized by work of adhesion $W_{a d}$. Within the range of retraction displacement of this work (less or equal to the punch radius), the stiffness of the system is insensitive toretraction displacement and depends only on the elasto-capillary number. In general, surface tension increases the slope of loaddisplacement curve or the stiffness of the system. For a given work of adhesion, the pull-off force (displacement) increases (decreases) with surface tension. Our results show that theapparent contact angleis not 90 degrees, as predicted by linear elastic theory, but varies from 20 to 180 degrees and is very sensitive to $\omega$ when it is near zero.This resultcan be potentially used to measure solid surface tension.

\section{Introduction}

Theindentation of a rigid flat circular punch into an elastic half space is a classicproblem in mechanics. It was first solved by Boussinesq[1]. His solution can be readily extended to determine the force required to retract the indenter when the indenter/substrate interface is adhesive[2-5], and this is a fundamental result in adhesion science. However, this force is a lower bound since it ignores the resistance due to elasto-capillarity, that is, it assumes that surface stress of the elastic substrate plays no role in resisting deformation. For soft materials, this assumption can cause substantial errors, asdemonstrated by recent experiments[6-9] and theories[10-13]. More references on the effect of surface stress on the mechanical behavior of soft materials can be found in a recent review by Style et al. [14]. In general, whether or not surface stresses are important depends on the elasto-capillary number $\omega$, defined as the ratio of the elasto-capillary length $\sigma / E$ to the characteristic length scale of the problem of interest. For the adhesion problem in this work, this length is $a$, the radius of the indenter. Thus, elastocapillaritycomes into play when the size of the indenter is small or when the substrate is very compliant. 
Another limitation to the Boussinesq's solution is that it assumes small strain linear elasticity (SSLE). Experiments havedemonstrated that large deformationsare often associated with elasto-capillary phenomenon $[7,9]$. Therefore, the Boussinesq solution is not expected to work for problems where $\omega \geq 1$

The adhesion problem considered in this work is closely related to adhesive contact mechanics of spheres and cylinders. Recently, several research groups have shown that surface stress can significantly affect the contact mechanics of these objects when $\omega \geq 1$ [15-20]. A simple way to model the mechanics of contact is to view the air gap between the contacting surfaces as an external tractionfree crack; the crack front being the contact line. The equilibrium position of the contact line is determined by setting the energy release rate of this crack to the work of adhesion, $\mathrm{W}_{\mathrm{ad}}$. It is interesting to note that the energy release rate of this crack can be deduced from the "Hertz" solution in which the stress singularity at the contact line is suppressed by enforcing continuity of slope $[15,16,19]$. Finally, it should be noted that the analyses in [15-20] are based on small strain linear elasticity (SSLE). One of the main objectives of this work is to study how theenergy release rate depends on the applied load and the surface stress for large deformation.

The plan of this paper is as follows. Section 2 states the problem andsection 3 describes the finite element method. A formula for calculation of energy release rate is derived in section 4 . Numerical resultsfor the effect of surface tension on the deformation field, energy release rate and pull-off behaviorare presented in section 5, and a discussion and summary are given in section 6 .

\section{Statement of problem}

\subsection{Problem definition}

The geometryof the problem under consideration is shown in Fig. 1A rigid flat circular punch of radius ais incontact with an elastic half space. We assume that contact is adhesive so the interface can support tension. The half space is assumed to be isotropic, homogeneous andincompressible, and its elasticity is described by a neo-Hookean material model.

To describe the mechanical behavior of the surface, we restrict our attention to soft materials such as polymer gels and elastomers, for which the surface can be considered to be isotropic. For these materials, the surface stress tensor $\Sigma=\sigma I_{s}$ is isotropic, where $I_{s}$ is the surface identity tensor, and $\sigma$ is a scalardefined as the surface tension. We further assume that $\sigma$ is constant, independent of surface strain. As noted by Gibbs [21], surface tension in solids need not be the same as surface energy. However, for hydrogels where the bulk of the surface material is liquid, the surface tension is expected to be constant with a value approximately equal to the surface energy. This statement is consistent with many experimental studies on soft hydrogels as well as soft elastomers[22-26]. Consistent with an adhesive interface, weimpose no-slip condition on the interface between punch and substrate. As noted in our previous work $[15,16]$, by this assumption we need not consider a separate interfacial tension in the contact region. 


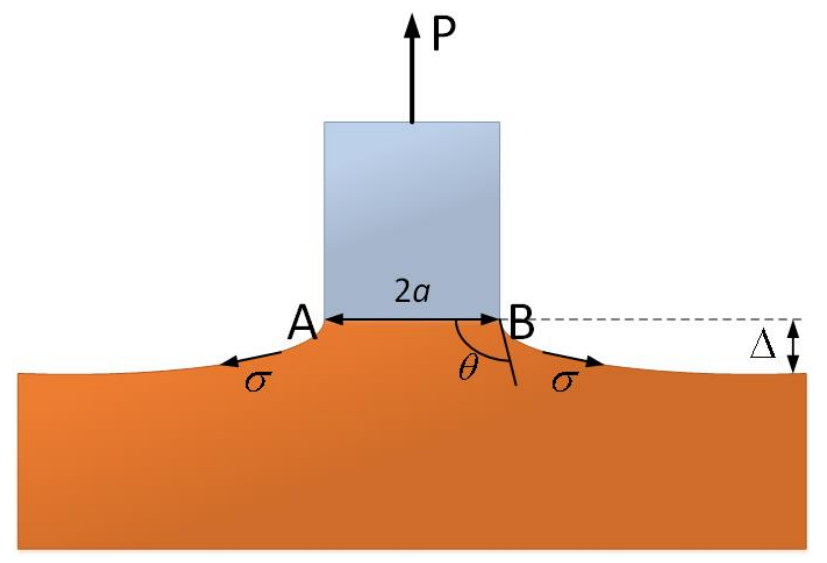

Fig.1 the contact between a rigid punch with radius $a$ and a semi-infinite elastic half-space under retraction $\mathrm{P}$. The retraction displacement is $\Delta$, and the contact angle between the substrate-air interface and the punch bottom is denoted by $\theta$.

\subsection{Limiting cases: Elasticity and Liquid-like limit}

Before tackling the general problem with surface tension, we consider two limits. In the first, elasticity dominates and surface tension is negligible $(\omega \rightarrow 0)$. In the second, the material is liquid-like with $\omega \rightarrow \infty$.

\subsubsection{Elastic limit, $\omega \rightarrow 0$}

In the limit of vanishing elasto-capillary number and assuming SSLE, the pressure distribution under the punch is[1]:

$$
p(r)=\frac{P}{2 \pi a^{2}}\left(1-\frac{r^{2}}{a^{2}}\right)^{-1 / 2}, \quad r<a
$$

where $P>0$ is the retraction load(see Fig. 1). It should be noted that Boussinesq's solution assumes frictionless contact. Fortunately, due to incompressibility, the interfacialsheartraction is also zero for no-slip contact. The relation between the retraction load $P$ and the indenter displacement $\Delta>0$ is given by

$$
P=\frac{8}{3} E a \Lambda
$$

where $E$ is the Young's modulus. Eqs. $(1,2)$ apply for both indentation and retraction as long as deformation is small. Fig. 2 illustrates that contact line can be viewed as the front of an external interface crack. Theenergy release rateGof this crackis [27]: 


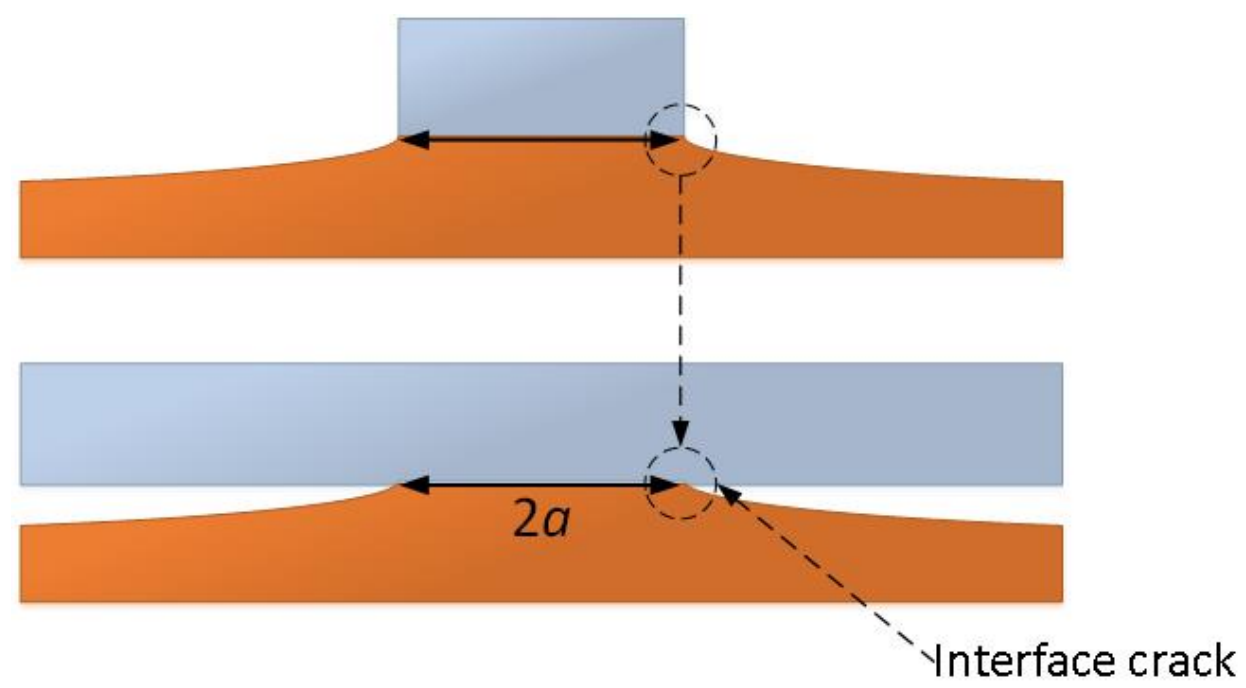

Fig. 2 During retraction, the edge of contact can be seen as the front of an external interface crack. This is because the indenter is rigid, so changing its shape outside the contact zone does not affect the stress and strain field in the substrate.

$$
G=\frac{3 P^{2}}{32 \pi E a^{3}}=\frac{2 E \Lambda^{2}}{3 \pi a}
$$

When the energy release rate is equal to the work of adhesion $W_{a d}$, the interface crack becomes unstable resulting in the punch suddenly jumping out of contact. The critical load and displacement associate with this instability are called pull-off force and displacement respectively and they are given by (3):

$$
\Delta_{o f f}^{(e)}=\sqrt{\frac{3 \pi a W_{a d}}{2 E}}, P_{o f f}^{(e)}=8 a \sqrt{\frac{\pi a E W_{a d}}{6}}
$$

where the superscript $(e)$ denotes the small strainelastic limit. Thus, the pull-off force/displacement can be used to determine the work of adhesion assuming that the modulus of the substrate is known.

For large deformation, exact results are not available. Kristnan et al.[27] have studied the punch problem using a finite element model without accounting for surface tension. These results are used to check the validity of our finite element model for the special case of $\omega \rightarrow 0$.

\subsubsection{Liquid-like limit $\omega \rightarrow \infty$}

In this limit, elasticity can be neglected in a large region surrounding the punch. We assume gravity is negligible, sothe pressure in the liquid-like "solid" is uniform and equals to atmosphere pressure and the free surface of the substrate is a zero mean curvature surface as shown in Fig. 3. 


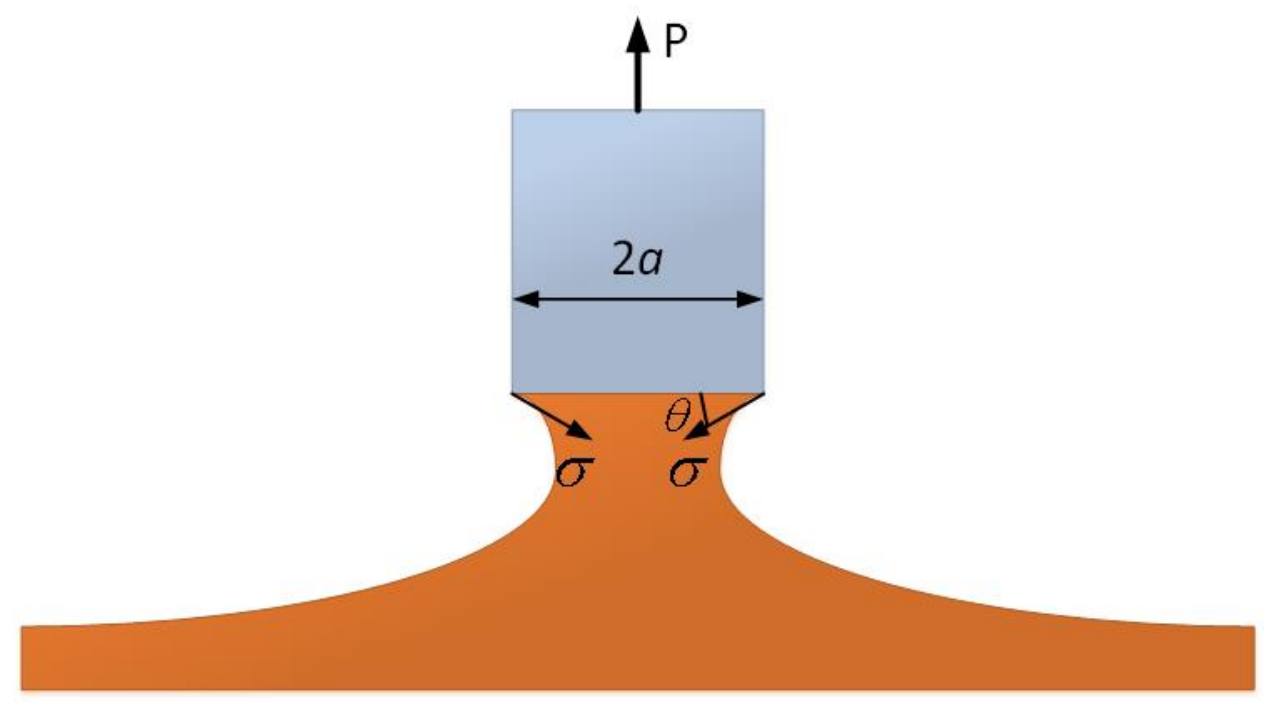

Fig. 3 Deformed shape of the substrate surface (zero mean curvature surface when gravity is neglected) in the liquid like limit, $\theta$ denotes the contact angle of the contact line.

In this limit, the contact angle $\theta$ at the punch edge (see Fig. 3 ) is determined by energy balance,which is equivalent toYoung-Dupre's equation assuming contact line is on lower surface ${ }^{1}[11]$ :

$$
\sigma(1+\cos \theta)=W_{a d}
$$

Force balance in z-direction (the direction of $P$ ) yields

$$
2 \pi a \sigma \sin \theta=P
$$

The pull-off load is determined by combining (5) and (6), i.e.,

$$
P_{o f f}^{(l)}=2 \pi a \sigma \sqrt{2 \frac{W_{a d}}{\sigma}-\left(\frac{W_{a d}}{\sigma}\right)^{2}}
$$

where the superscript I denotes the liquid limit.In this limit, large contact angle ( $\theta \approx \pi$ )implies small deformation, for this case, $W_{a d} / \sigma \square 1$ so (7a) reduces to

$$
P_{o f f}^{(l)} \approx 2 \pi a \sqrt{2 \sigma W_{a d}} . \quad W_{a d} / \sigma \square 1
$$

Finally, it should be noted that the displacement of the punch is not defined in the liquid like limit for an infinite substrate. Indeed, the deformed substrate has a zero mean curvature surface, and surface displacement increases logarithmically with the size of the substrate.

\section{Finite Element (FE) Method}

\footnotetext{
${ }^{1}$ In practice, (5) is a lower bound since the contact angle can be anywhere between what is given by (5) and $3 \pi / 2$ because perfectly sharp corner does not exist.
} 
Our FE model is shown schematically in Fig. 4. A commercial FEM software, ABAQUS ${ }^{\circledR}$, is used to compute the deformation of the substrate which is assumed to be an incompressible neo-hookean solid with strain energy density $W$ given by

$$
W=\frac{E}{6}\left(l_{1}-3\right)
$$

where $I_{1}=\sum_{i=1}^{3} \lambda_{i}^{2}$ is the first invariant of the right Cauchy-Green strain tensor with the principle stretches denoted by $\lambda_{i}$. The built-in Neo-Hookean function in ABAQUS is selected to model this material behavior. In our model, all lengths are normalized by $a$ and retractionload $P$ is normalized by its corresponding value in the elastic limit with displacement equal to $a$ (see eq.2), that is

$$
\bar{P}=3 P /\left(8 E a^{2}\right), \bar{\Lambda}=\Delta / a, \bar{r}=r / a
$$

With this normalization, the dimensionless solution depends only on a single dimensionless parameter, the elasto-capillary number $\omega=\sigma /(E a)$. The infinite cross-section of the substrate is approximated by a square with a length of 100 dimensionless units (the contact extends over a radial distance ofone). We have checked that increasing the size of the square does not affect the solution of the stress and deformation fields near the punch. On boundary 1 (see Fig. 4), a given displacement is prescribed in the z-direction while the radial displacement is fixed to be zero to enforce the no-slip condition. No radial displacement is allowed on boundaries 2 and 4 while the $z$ - displacement and shear traction are both zero on boundary 3 . On boundary 5 , a user-defined surface element (UEL) is used to model surface tension. Details about the implementation of this surface element in ABAQUS can be found in the work of Xu et al.[28]. The built-in element CAX4RH is selected as bulk element type. We monitor the distortion of elements and, when the distortion is too large, a re-meshing python script is run to generate a new mesh on the deformed configuration to ensure proper convergence and accuracy of calculation.

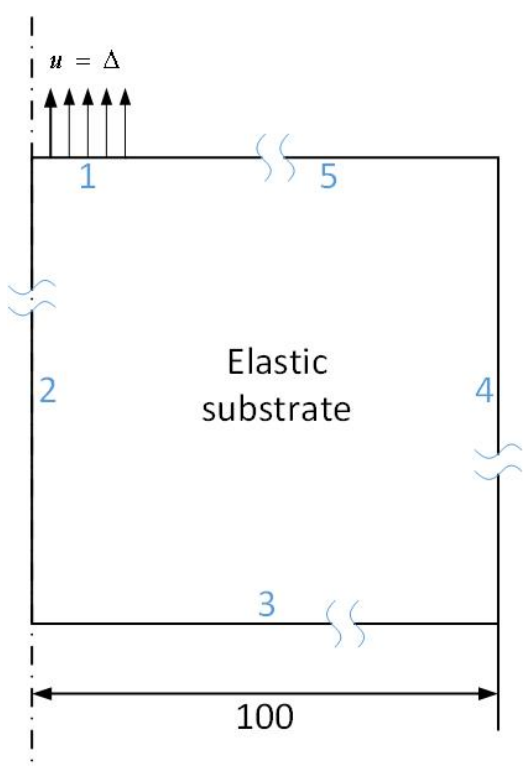


Fig. 4 A schematic of ourmodel used in finite element calculation. All length are normalized by the punch radius. The elastic half-space is approximated by a square with length equal to 100 unit. $d$

\section{Calculation of energy release rate $G$}

Our main objective is to determine the relation between the load and displacement during retraction. The pull-off load and displacementare determined by the energy balance equation, i.e., the energy release rate of the interface crack is equal to the work of adhesion,

$$
G=W_{a d}
$$

In the literature, calculation of energy release rate usually requires local stress or displacement field near crack tip. Because of the high gradients at the crack tip, these quantities are much more susceptible to error in comparison with global quantities like indenter load, indenter displacement and total elastic energy. Here we introduce a formulation which takes advantage of this fact.

In a displacement controlled test, the energy release rate is given by

$$
G=\left.\frac{\partial \Gamma}{\partial A}\right|_{\Delta}
$$

where $\Gamma$ is thetotal energy of the substrate and $A=\pi a^{2}$ is the contact area. The normalized total energy $\bar{\Gamma}=\Gamma /\left(\frac{8}{3} E a^{3}\right)$ is the work done by the normalized load acting on the normalized displacement, i.e.,

$$
\bar{\Gamma}=\int_{0}^{\bar{A}} \bar{P}\left(\bar{\Delta}^{\prime}, \omega\right) d \bar{\Delta}^{\prime}
$$

Dimensional consideration imply that the normalizedtotal energy $\bar{\Gamma}$ and normalized load $\bar{P}$ is a function of $\bar{\Delta}$ and $\omega$ only. Since both $\bar{\Delta}$ and $\omega$ in (11) contain $a=\sqrt{A / \pi}$, the derivative of $\Gamma$ in (11) consists of three terms given by(see Supplementary material (SM) for details)

$$
G=\frac{4 E a}{\pi}\left(\bar{\Gamma}(\bar{\Delta}, \omega)-\frac{1}{3} \frac{\partial(\bar{\Gamma}(\bar{\Delta}, \omega))}{\partial(\bar{\Delta})} \bar{\Delta}-\frac{1}{3} \frac{\partial(\bar{\Gamma}(\bar{\Delta}, \omega))}{\partial(\omega)} \omega\right)
$$

After normalizingenergy release rate by $\frac{4 E a}{\pi}$, i.e., $\bar{G}=\frac{\pi G}{4 E a}$, Eq. (13) becomes:

$$
\bar{G}=\frac{\pi G}{4 E a}=\bar{\Gamma}(\bar{\Lambda}, \omega)-\frac{1}{3} \frac{\partial \bar{\Gamma}(\bar{\Lambda}, \omega)}{\partial \bar{\Lambda}} \bar{\Lambda}-\frac{1}{3} \frac{\partial \bar{\Gamma}(\bar{\Lambda}, \omega)}{\partial \omega} \omega
$$

Eq. (14) allows us to evaluate the energy release rate using global quantities such as strain energy and its variations with the normalized displacement and elasto-capillary number. Specifically, the first term of Eq. (14) is computed by numerical integration using Eq. (12) and the last two terms are evaluated by a first order central finite difference method. In post-processing, the sum of $z$-direction reaction force on 
boundary 3 is used to compute the normal load in Eq. 12. Note that while(13) is strictly valid for a semiinfinite substrate, a correction term needs to be added when a finite substrate is considered (see supplementary information).

\section{Numerical Results}

The local surface displacement profiles for different retraction displacement are shown in Figure 5 for fivedifferent elasto-capillary numbers. In the absence of surface tension ( $\omega=0)$, SSLE theory predicts that the contact angle should be exactly $90^{\circ}$; however, the free surface actuallydeforms into a neck like region with the local contact angle close to zero. As shown in Fig. 5, the neck becomes more severe as retraction increases from 0.1 to 1 . This result is consistent with the earlier analysis by Krishnan et al.[27] where $\omega=0$. As shown in Fig. 5 , the local kink at the contact line flattened as elasto-capillary number increases, consistent with the intuition that surface tension always mitigate local curvature.

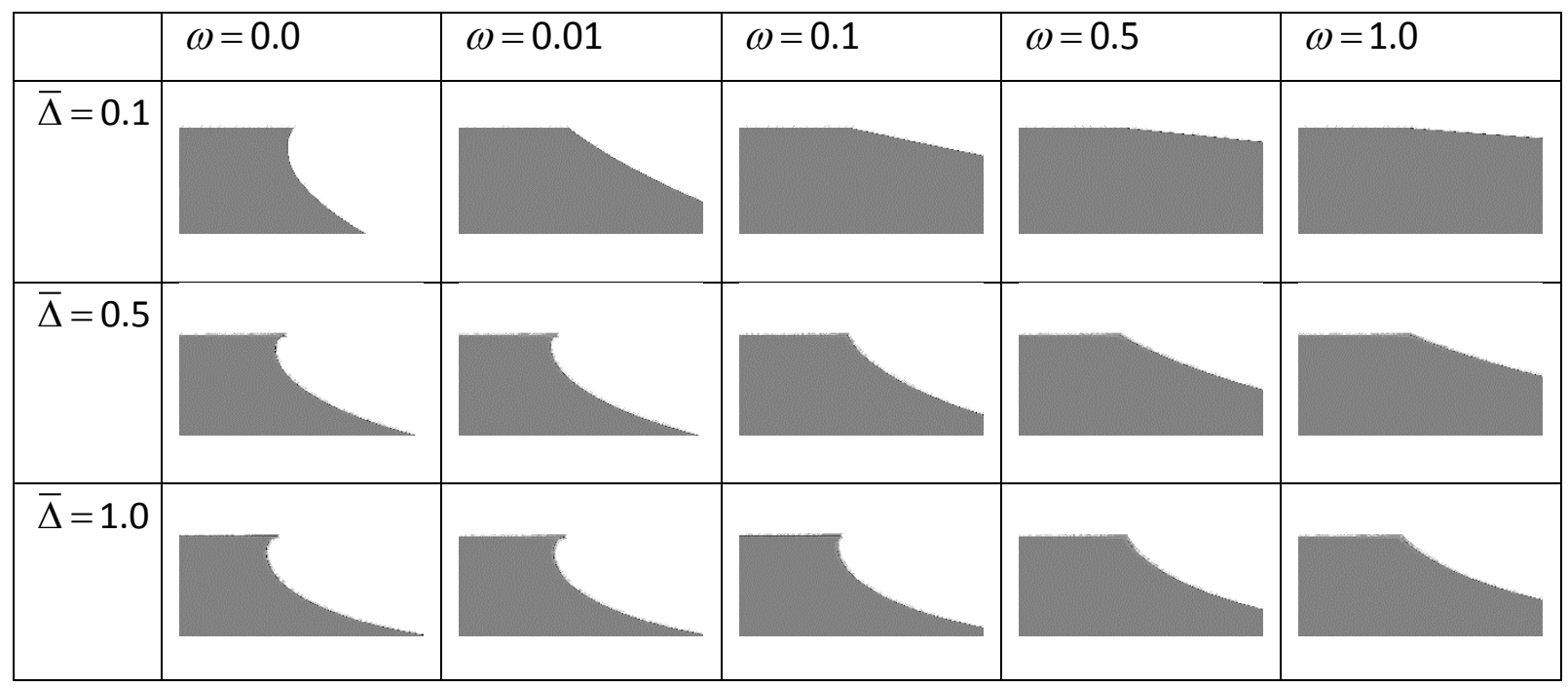

Fig. 5 Near edge deformation field for retraction of punch. The gray part is the deformed shape of the substrate near the punch edge. With increase of elasto-capillary number, the substrate becomes more flat and the local contact angle becomes larger.

The stiffness of the system kisdefined as the ratio of the indenter force overthe indenter displacement. In linear theory, $k$ depends on $a, \omega$ and is independent of $\Delta$. However, for large deformation or nonlinear material behavior, this need not be the case. Because the normalized load is a function only of $\bar{\Delta}$ and $\omega$, it is convenient to define a normalized stiffness $\bar{k}=\bar{P} / \bar{\Delta}$, so

$$
k=P / \Delta=\frac{8 E a}{3} \bar{k}(\bar{\Delta}, \omega)
$$

Surprisingly, our FEM results in Fig. 6 show thatthe force versus indentation displacement curve is practically linear for $\Delta \leq a$, that is, the normalized stiffness is a function of $\omega$ only in this range, i.e.,

$$
\bar{k}(\bar{\Delta}, \omega) \square \bar{k}(\omega), \bar{\Delta} \leq 1
$$


Note that the normalized stiffness increases with the elasto-capillary number. Also, in Fig. 6, we compared our resultswith the SSLE results of Wang et al. [29] which are shown as red lines. Our result shows that SSLE underestimates the stiffness and the error increases with increasing elasto-capillary number.One source of error of SSLE comes from the fact that the contact angle at the contact edge cannot be correctly predicted by linear theory.

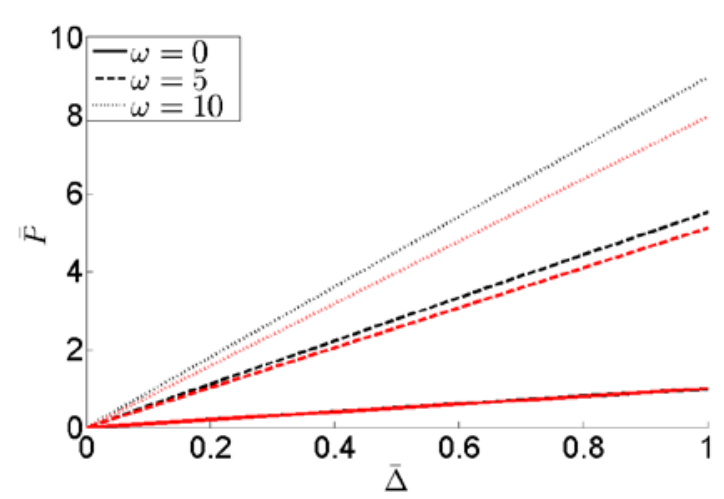

(a)

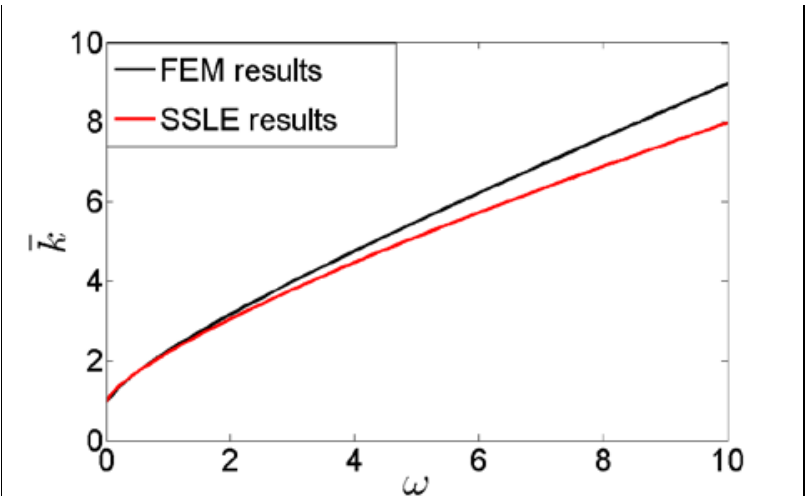

(b)

Fig. 6 (a) Normalized load-displacement relation for retraction of punch for three different elastocapillary numbers. The three red lines are the corresponding SSLE results given by Wang et al [29]. (b) Comparison of stiffness with SSLE results. SSLE tends to underestimate the stiffness.

Results for thenormalized energy release rateare shownin Fig. 7(a,b).

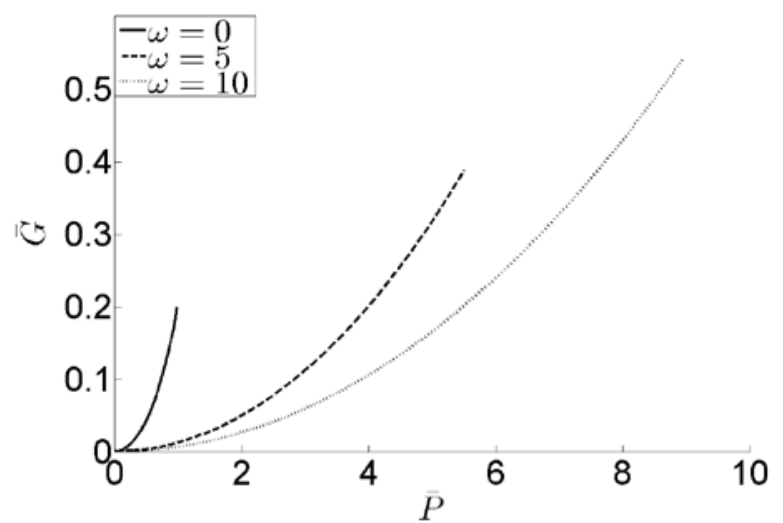

(a)

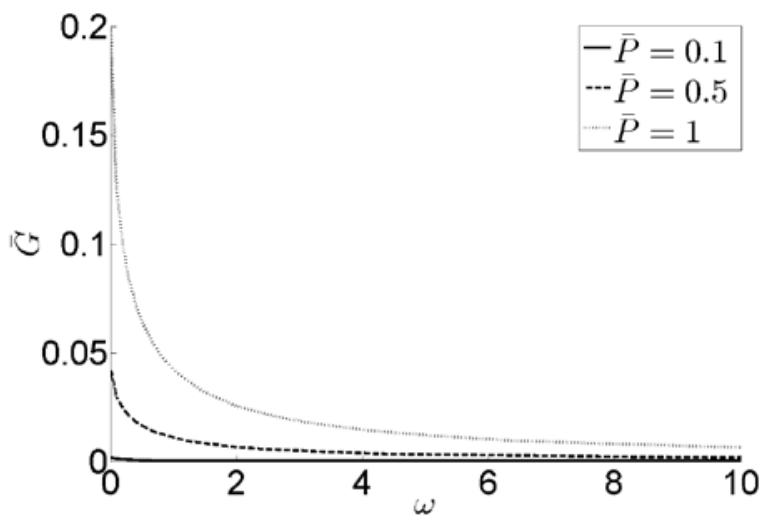

(b)

Fig. 7(a) Normalized energy release rate vs normalized load for different values of elasto-capillary number (b) Normalized energy release rate vs elaso-capillary number for different normalized load.

Physically, one expects that, for a fixed elasto-capillary number $\omega$, a higher external load will provide a higher driving force to propagate the crack and hence corresponds to a higher energy release rate, as shown in Fig. 7 (a). On the other hand, the presence of surface stress tends to close the crack as 
suggested by a previous calculation[30]. Therefore, for a fixed external load, the energy release rate will decrease with elasto-capillary number as shown in Fig. 7 (b).

Once the energy release rate is obtained, the pull-off load and displacement can be determined using the energy balance equation $G=W_{a d}$. Fig $8(\mathrm{a}, \mathrm{b})$ plots the pull-off load and displacement normalized by their corresponding value in the elasticity dominated limit (Eq. (4)) to highlight the effect of surface tension.

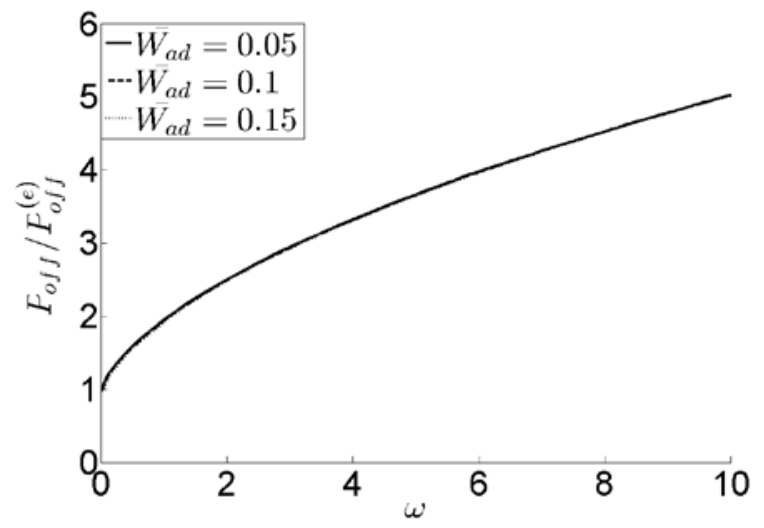

(a)

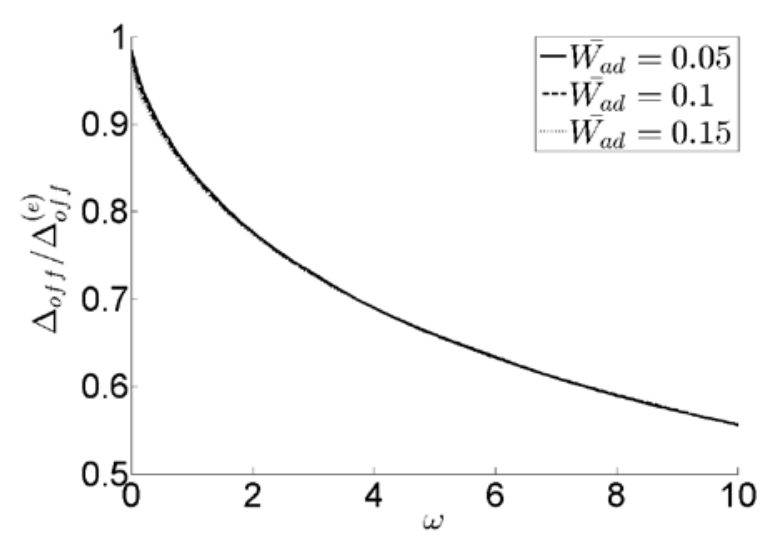

(b)

Fig. 8 (a) normalized pull-off load vs elasto-capillary number (b) normalized pull-off displacement vs elasto-capillary number. The curves with different normalized work of adhesion $\overline{W_{a d}}=\frac{\pi W_{a d}}{4 E a}$ essentially lie on top of each other.

Fig. $8(a, b)$ show an important result, the curves for different normalized $\overline{W_{a d}}=\frac{\pi W_{a d}}{4 E a}$ lie on top of each other.Thus, the normalized pull-off load/displacement depend only on the elasto-capillary number, i.e.,

$$
\begin{aligned}
& P_{o f f}=8 a \sqrt{\frac{\pi a E W_{a d}}{6}} \widehat{P_{o f f}}(\omega) \\
& \Delta_{o f f}=\sqrt{\frac{3 \pi a W_{a d d}}{2 E}} \widehat{\Delta_{o f f}}(\omega)
\end{aligned}
$$

Note that the pull-off load increases with the elasto-capillary number while the pull-off displacement decreases with it, consistent with the intuition that surface tension opposes deformation.These results are also consistent with previous work for spherical indenter [16] and 2-D cylindrical indenter [15]. In the SM, we show that $(17 a, b)$ is exactif the stiffness of the systemdepends only on $\omega$.

In the range of our calculation, $0 \leq \omega \leq 10, P_{\text {off }}(\omega)$ and $\Delta_{\text {off }}(\omega)$ can be fitted accurately by the following expressions: 


$$
\begin{aligned}
& P_{\text {off }}(\omega)=\frac{2.4934 \omega+1.54 \omega^{1 / 2}+1}{1.6244 \omega^{1 / 2}+1} \\
& \triangle_{\text {off }}(\omega)=\frac{0.5035 \omega^{1 / 2}+1}{0.1678 \omega+0.6225 \omega^{1 / 2}+1}
\end{aligned}
$$

Since gravity is ignored in our calculation, the indenter displacement is not defined (or depend on the scale of the substrate) in the liquid limit, so these formulaeshould be used within the range of $0 \leq \omega \leq 10$.

\section{Discussion and summary}

\subsection{Discussion}

Our finite element results shows that the indentation load-displacement curve is practically linear for $-1 \leq \bar{\Delta} \leq 1$, as a result, thenormalized stiffness is a function of elasto-capillary number alone. This result suggested that solid surface tension can be measured by evaluating the slope of the loading curve if one can independently measure the elastic modulus of the solid.In many applications, the Elasto-capillary number is quite small. For example, the elasto-capillary number of a punch with a $1 \mathrm{~mm}$ diameter and a soft silicone gel with modulus on the order of $1 \mathrm{KPa}$ [7] is about 0.1 . For these cases, Fig. $6(\mathrm{~b})$ shows that thenormalized stiffness is not so sensitive toelasto-capillary number, as a result,the change in stiffness due to surface tension may not be significant enough to be detected. However, Fig. 5 shows that the deformation near the contact line is very sensitive to $\omega$ when it is small. Hence, for small $\omega$, an alternative way to determinesubstrate surface tension is to measure the "apparent contact angle" $\theta$. Fig.9plots the apparent contact angle as a function of the elasto-capillary number for $\bar{\Delta}=1.0$. Note that $\theta$ increases very rapidly for small $\omega$. On the other hand, for an ultra-soft hydrogel with modulus on the order of 10Pa [31], $\omega$ is about 10, for this case the substrate stiffnessshould be used to determine the surface tension.

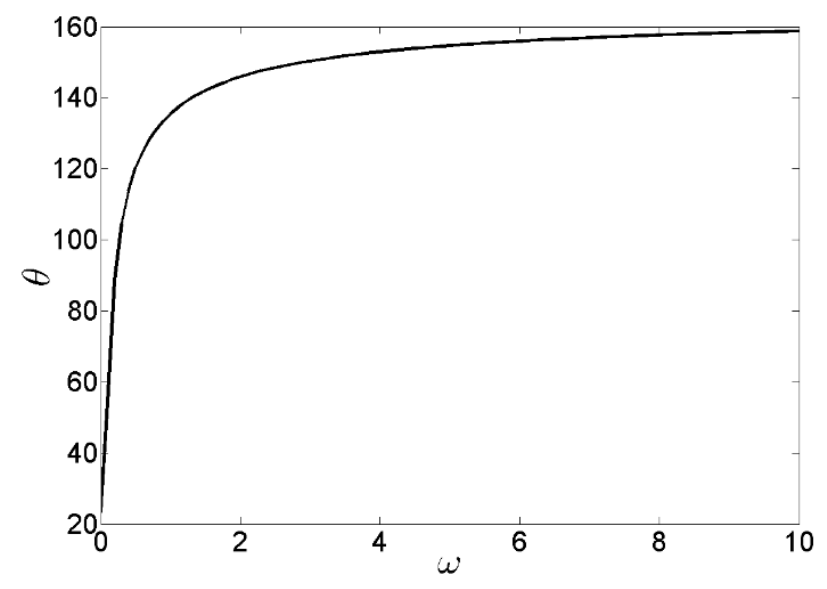


Fig. 9 Apparent contact angle vs elasto-capillary number for $\bar{\Delta}=1.0$

An important subset of problem of practical interest we did not fully address here is the effect of surface tension on indentation, where $P<0$. This is because indentation can cause the side wall of the indenter to come into contact with the surface of the substrate, as shown by our FE calculations which is included in the SM. These FE results show that as long as the amount of indentation does not exceed the contact radius and the elasto-capillary number is greater or equal to 0.1 , sidewall contact does not occur and Eq. (15) holds for both indentation and retraction. In general, side contact will affect the indenter force even if frictionless boundary condition were imposed between the sidewall and substrate. Of course, in reality, some friction will exist, and this will provide additional resistance to indentation and further complicates the analysis.

\subsection{Summary}

Finite element simulationsare used to investigate the effects of surface tension on the adhesive contact between a rigid flat circular punch and an elastic substrate. The elasto-capillary number $\omega=\frac{\sigma}{E a}$ is used to quantify surface tension effects. Our calculation showsthat surface tension can significantly mitigate the local concentration of curvature near the edge of the punch. This change of local deformation is very sensitive to elasto-capillary number when it is small. For fixed indentation load, it is shown that energy release rate goes down when $\omega$ increases which suggests that the presence of surface tension makes the propagation of an interface crack more difficult. An examination of pull-off behavior shows that a higher external load but a smaller displacement is required to pull the punch out of contact when there is surface tension. Finally, we propose that the loading curve slope and the "contact angle" at the punch edge for fixed displacement is a function of elasto-capillary number only and may be used as a method to measure the substrate surface tension. We also provide approximate expressions for the pull-off load and displacement.

In a previous work of a rigid sphere in contact with an elastic substrate, Karpitscha et al.[32] found that the local contact angle is always determined by Young's equation, assuming surface energies equal to surface tensions. Our pull-off results are consistent with their theory even though the geometry is different (see supplementary information).

There are limitations in our work. We have not been able to consider very large deformation where $\Delta>a$. Such large deformation requires a considerable amount of remeshing which affects the accuracy of the FE solution. We assume the surface stress is isotropic and can be represented by a constant surface tension $\sigma$ independent of surface strain, so our surface model can only be applied to a restricted class of soft materials. It is also assumed that the contact line is perfectly circular whereas in reality the presence of defect may cause small error when compared with theory. Finally, we assume adhesion is sufficiently strong so that the interface does not slip before it fails.

\section{Acknowledgement}


All three authors acknowledge the support by the U.S. Department of Energy, Office of Basic Energy Sciences, Division of Materials Sciences and Engineering under Award DE-FG02-07ER46463. We thanks an anomalous reviewer for his/her comments.

\section{References}

[1] J. Boussinesq, Application des potentiels à l'étude de l'équilibre et du mouvement des solides élastiques principalement au calcul des déformations et des pressions que produisent, dans ces solides, des, (1885).

[2] K.L. Johnson, Contact Mechanics, Cambridge University Press, 1987. http://www.cambridge.org/us/academic/subjects/engineering/solid-mechanics-andmaterials/contact-mechanics (accessed October 14, 2014).

[3] K.L. Johnson, K. Kendall, A.D. Roberts, Surface Energy and the Contact of Elastic Solids, Proc. R. Soc. A Math. Phys. Eng. Sci. 324 (1971) 301-313. doi:10.1098/rspa.1971.0141.

[4] B.. Derjaguin, V.. Muller, Y.. Toporov, Effect of contact deformations on the adhesion of particles, J. Colloid Interface Sci. 53 (1975) 314-326. doi:10.1016/0021-9797(75)90018-1.

[5] D. Maugis, Adhesion of spheres: The JKR-DMT transition using a dugdale model, J. Colloid Interface Sci. 150 (1992) 243-269. doi:10.1016/0021-9797(92)90285-T.

[6] K.E. Jensen, R. Sarfati, R.W. Style, R. Boltyanskiy, A. Chakrabarti, M.K. Chaudhury, et al., Wetting and phase separation in soft adhesion., Proc. Natl. Acad. Sci. U. S. A. 112 (2015) 14490-4. doi:10.1073/pnas.1514378112.

[7] R.W. Style, C. Hyland, R. Boltyanskiy, J.S. Wettlaufer, E.R. Dufresne, Surface tension and contact with soft elastic solids., Nat. Commun. 4 (2013) 2728. doi:10.1038/ncomms3728.

[8] B. Andreotti, O. Baeumchen, F. Boulogne, K.E. Daniels, E.R. Dufresne, H. Perrin, et al., Solid Capillarity: When and How does Surface Tension Deform Soft Solids?, (2015) 5. http://arxiv.org/abs/1512.08705 (accessed February 16, 2016).

[9] A. Chakrabarti, M.K. Chaudhury, Elastocapillary interaction of particles on the surfaces of ultrasoft gels: a novel route to study self-assembly and soft lubrication., Langmuir. 30 (2014) 4684-93. doi:10.1021/la5007988.

[10] D. Tabor, Surface forces and surface interactions, J. Colloid Interface Sci. 58 (1977) 2-13. doi:10.1016/0021-9797(77)90366-6.

[11] C.-Y. Hui, A. Jagota, Deformation near a liquid contact line on an elastic substrate, Proc. R. Soc. A Math. Phys. Eng. Sci. 470 (2014) 20140085-20140085. doi:10.1098/rspa.2014.0085.

[12] A. Jagota, D. Paretkar, A. Ghatak, Surface-tension-induced flattening of a nearly plane elastic solid, Phys. Rev. E. 85 (2012) 51602. doi:10.1103/PhysRevE.85.051602.

[13] X. Xu, A. Jagota, S. Peng, D. Luo, M. Wu, C.-Y. Hui, Gravity and surface tension effects on the shape change of soft materials., Langmuir. 29 (2013) 8665-74. doi:10.1021/la400921h.

[14] R.W. Style, A. Jagota, C.-Y. Hui, E.R. Dufresne, Elastocapillarity: Surface Tension and the Mechanics of Soft Solids, (2016). http://arxiv.org/abs/1604.02052 (accessed April 15, 2016).

[15] T. Liu, A. Jagota, C.-Y. Hui, Adhesive contact of a rigid circular cylinder to a soft elastic substrate the role of surface tension, Soft Matter. (2015). doi:10.1039/C5SM00008D. 
[16] C.-Y. Hui, T. Liu, T. Salez, E. Raphael, A. Jagota, Indentation of a rigid sphere into an elastic substrate with surface tension and adhesion, Proc. R. Soc. A Math. Phys. Eng. Sci. 471 (2015) 20140727-20140727. doi:10.1098/rspa.2014.0727.

[17] J.M. Long, G.F. Wang, X.Q. Feng, S.W. Yu, Two-dimensional Hertzian contact problem with surface tension, Int. J. Solids Struct. 49 (2012) 1588-1594. doi:10.1016/j.ijsolstr.2012.03.017.

[18] J.M. Long, G.F. Wang, Effects of surface tension on axisymmetric Hertzian contact problem, Mech. Mater. 56 (2013) 65-70. doi:10.1016/j.mechmat.2012.09.003.

[19] X. Gao, F. Hao, Z. Huang, D. Fang, Mechanics of adhesive contact at the nanoscale: The effect of surface stress, Int. J. Solids Struct. 51 (2014) 566-574. doi:10.1016/j.ijsolstr.2013.10.017.

[20] X. Gao, F. Hao, D. Fang, Z. Huang, Boussinesq problem with the surface effect and its application to contact mechanics at the nanoscale, Int. J. Solids Struct. 50 (2013) 2620-2630. doi:10.1016/j.ijsolstr.2013.04.007.

[21] C.-Y. Hui, A. Jagota, Surface tension, surface energy, and chemical potential due to their difference., Langmuir. 29 (2013) 11310-6. doi:10.1021/la400937r.

[22] R. Style, R. Boltyanskiy, Y. Che, J. Wettlaufer, L.A. Wilen, E. Dufresne, Universal Deformation of Soft Substrates Near a Contact Line and the Direct Measurement of Solid Surface Stresses, Phys. Rev. Lett. 110 (2013) 66103. doi:10.1103/PhysRevLett.110.066103.

[23] S. Mora, T. Phou, J.-M. Fromental, L.M. Pismen, Y. Pomeau, Capillarity Driven Instability of a Soft Solid, Phys. Rev. Lett. 105 (2010) 214301. doi:10.1103/PhysRevLett.105.214301.

[24] S. Mora, M. Abkarian, H. Tabuteau, Y. Pomeau, M. Biot, M. Kücken, et al., Surface instability of soft solids under strain, Soft Matter. 7 (2011) 10612. doi:10.1039/c1sm06051a.

[25] S. Mora, C. Maurini, T. Phou, J.-M. Fromental, B. Audoly, Y. Pomeau, Solid Drops: Large Capillary Deformations of Immersed Elastic Rods, Phys. Rev. Lett. 111 (2013) 114301. doi:10.1103/PhysRevLett.111.114301.

[26] X. Xu, A. Jagota, D. Paretkar, C.-Y. Hui, Surface tension measurement from indentation of clamped thin films, Soft Matter. 12 (2016) 5121-5126. doi:10.1039/C6SM00584E.

[27] V.R. Krishnan, C.-Y. Hui, Large deformation of soft elastic materials in adhesive contact with a rigid cylindrical flat punch, Soft Matter. 4 (2008) 1909. doi:10.1039/b804340j.

[28] X. Xu, A. Jagota, C.-Y. Hui, Effects of surface tension on the adhesive contact of a rigid sphere to a compliant substrate., Soft Matter. 10 (2014) 4625-32. doi:10.1039/c4sm00216d.

[29] G.F. Wang, X.R. Niu, Nanoindentation of soft solids by a flat punch, Acta Mech. Sin. 31 (2015) 531-535. doi:10.1007/s10409-015-0440-7.

[30] T. Liu, R. Long, C.-Y. Hui, The energy release rate of a pressurized crack in soft elastic materials: effects of surface tension and large deformation., Soft Matter. 10 (2014) 7723-9. doi:10.1039/c4sm01129e.

[31] A. Chakrabarti, M.K. Chaudhury, Direct measurement of the surface tension of a soft elastic hydrogel: exploration of elastocapillary instability in adhesion., Langmuir. 29 (2013) 6926-35. doi:10.1021/la401115j.

[32] S. Karpitschka, L. van Wijngaarden, J.H. Snoeijer, Surface Tension regularizes the Crack Singularity of Adhesive Contacts, (2015) 5. http://arxiv.org/abs/1508.03588 (accessed February 16, 2016).

[33] C.-Y. Hui, A. Jagota, Deformation near a liquid contact line on an elastic substrate, Proc. R. Soc. A 
Math. Phys. Eng. Sci. 470 (2014) 20140085-20140085. doi:10.1098/rspa.2014.0085. 